\title{
A Third-Order Differential Equation and Starlikeness of a Double Integral Operator
}

\author{
Rosihan M. Ali, ${ }^{1}$ See Keong Lee, ${ }^{1}$ K. G. Subramanian, ${ }^{1}$ \\ and A. Swaminathan ${ }^{2}$ \\ ${ }^{1}$ School of Mathematical Sciences, Universiti Sains Malaysia (USM), Penang 11800, Malaysia \\ ${ }^{2}$ Department of Mathematics, Indian Institute of Technology Roorkee, Roorkee 247 667, India
}

Correspondence should be addressed to Rosihan M. Ali, rosihan@cs.usm.my

Received 19 August 2010; Revised 2 January 2011; Accepted 10 January 2011

Academic Editor: Jean Pierre Gossez

Copyright (C) 2011 Rosihan M. Ali et al. This is an open access article distributed under the Creative Commons Attribution License, which permits unrestricted use, distribution, and reproduction in any medium, provided the original work is properly cited.

Functions $f(z)=z+\sum_{2}^{\infty} a_{n} z^{n}$ that are analytic in the unit disk and satisfy the differential equation $f^{\prime}(z)+\alpha z f^{\prime \prime}(z)+\gamma z^{2} f^{\prime \prime \prime}(z)=g(z)$ are considered, where $g$ is subordinated to a normalized convex univalent function $h$. These functions $f$ are given by a double integral operator of the form $f(z)=\int_{0}^{1} \int_{0}^{1} G\left(z t^{\mu} s^{v}\right) t^{-\mu} s^{-v} d s d t$ with $G^{\prime}$ subordinated to $h$. The best dominant to all solutions of the differential equation is obtained. Starlikeness properties and various sharp estimates of these solutions are investigated for particular cases of the convex function $h$.

\section{Introduction}

Let $\mathcal{A}$ denote the class of all analytic functions $f$ defined in the open unit $\operatorname{disk} U:=\{z \in \mathbb{C}$ : $|z|<1\}$ and normalized by the conditions $f(0)=0, f^{\prime}(0)=1$. Further, let $\mathcal{S}$ be the subclass of $A$ consisting of univalent functions, and let $\mathcal{S}^{*}$ be its subclass of starlike functions. A starlike function $f$ is characterized analytically by the condition $\operatorname{Re}\left(z f^{\prime}(z) / f(z)\right)>0$ in $U$, that is, the domain $f(U)$ is starlike with respect to origin. For two functions $f(z)=z+a_{2} z^{2}+\cdots$ and $g(z)=z+b_{2} z^{2}+\cdots$ in $\mathcal{A}$, the Hadamard product (or convolution) of $f$ and $g$ is the function $f * g$ defined by

$$
(f * g)(z)=z+\sum_{n=2}^{\infty} a_{n} b_{n} z^{n}
$$

For $f$ and $g$ in $\mathcal{A}$, a function $f$ is subordinate to $g$, written as $f(z) \prec g(z)$, if there is an analytic function $w$ satisfying $w(0)=0$ and $|w(z)|<1$, such that $f(z)=g(w(z)), z \in U$. 
When $g$ is univalent in $U$, then $f$ is subordinated to $g$ which is equivalent to $f(U) \subset g(U)$ and $f(0)=g(0)$.

In a recent paper, Miller and Mocanu [1] investigated starlikeness properties of functions $f$ defined by double integral operators of the form

$$
f(z)=\int_{0}^{1} \int_{0}^{1} W(s, t, z) d s d t
$$

In this paper, conditions on a different kernel $W$ are investigated from the perspective of starlikeness. Specifically, we consider functions $f \in \mathcal{A}$ given by the double integral operator of the form

$$
f(z)=\int_{0}^{1} \int_{0}^{1} G\left(z t^{\mu} s^{v}\right) t^{-\mu} s^{-v} d s d t
$$

In this case, it follows that

$$
f^{\prime}(z)=\int_{0}^{1} \int_{0}^{1} g\left(z t^{\mu} s^{v}\right) d s d t
$$

where $G^{\prime}(z)=g(z)$. Further, the function $f$ satisfies a third-order differential equation of the form

$$
f^{\prime}(z)+\alpha z f^{\prime \prime}(z)+\gamma z^{2} f^{\prime \prime \prime}(z)=g(z)
$$

for appropriate parameters $\alpha$ and $\gamma$. The investigation of such functions $f$ can be seen as an extension to the study of the class

$$
R(\alpha, h)=\left\{f \in \mathcal{A}: f^{\prime}(z)+\alpha z f^{\prime \prime}(z) \prec h(z), z \in U\right\} .
$$

The class $R(\alpha, h)$ or its variations for an appropriate function $h$ have been investigated in several works; see, for example, [2-10] and more recently [11, 12].

\section{Results on Differential Subordination}

We first recall the definition of best dominant solution of a differential subordination.

Definition 2.1 ((dominant and best dominant) [13]). Let $\Psi: \mathbb{C}^{3} \times U \rightarrow \mathbb{C}$, and let $h$ be univalent in $U$. If $p$ is analytic in $U$ and satisfies the differential subordination

$$
\Psi\left(p(z), z p^{\prime}(z), z^{2} p^{\prime \prime}(z)\right) \prec h(z)
$$

then $p$ is called a solution of the differential subordination. A univalent function $q$ is called a dominant if $p<q$ for all $p$ satisfying (2.1). A dominant $\tilde{q}$ that satisfies $\tilde{q}<q$ for all dominants $q$ of (2.1) is said to be the best dominant of (2.1). 
In the following sequel, we will assume that $h$ is an analytic convex function in $U$ with $h(0)=1$. For $\alpha \geq \gamma \geq 0$, consider the third-order differential equation

$$
f^{\prime}(z)+\alpha z f^{\prime \prime}(z)+\gamma z^{2} f^{\prime \prime \prime}(z)=g(z), \quad g(z) \prec h(z) .
$$

We will denote the class consisting of all solutions $f \in \mathcal{A}$ as $R(\alpha, \gamma, h)$, that is,

$$
R(\alpha, \gamma, h)=\left\{f \in \mathcal{A}: f^{\prime}(z)+\alpha z f^{\prime \prime}(z)+\gamma z^{2} f^{\prime \prime \prime}(z) \prec h(z), z \in U\right\}
$$

Let

$$
\mu=\frac{(\alpha-\gamma)-\sqrt{(\alpha-\gamma)^{2}-4 \gamma}}{2}, \quad v+\mu=\alpha-\gamma, \mu v=\gamma
$$

The discriminant is denoted by $\Delta:=(\alpha-\gamma)^{2}-4 \gamma$. Note that $\operatorname{Re} \mu \geq 0$ and $\operatorname{Re} v \geq 0$.

We will rewrite the solution of

$$
f^{\prime}(z)+\alpha z f^{\prime \prime}(z)+\gamma z^{2} f^{\prime \prime \prime}(z)=g(z)
$$

in its equivalent integral form

$$
f^{\prime}(z)=\int_{0}^{1} \int_{0}^{1} g\left(z t^{\mu} s^{v}\right) d s d t
$$

It follows from relations (2.4) that

$$
\begin{aligned}
g(z) & =f^{\prime}(z)+(\mu(1+v)+v) z f^{\prime \prime}(z)+\mu v z^{2} f^{\prime \prime \prime}(z) \\
& =v z^{1-1 / v}\left(\mu z^{1+1 / v} f^{\prime \prime}(z)+z^{1 / v} f^{\prime}(z)\right)^{\prime} \\
& =v z^{1-1 / v}\left(\mu z^{1+1 / v-1 / \mu}\left(z^{1 / \mu} f^{\prime}(z)\right)^{\prime}\right)^{\prime} .
\end{aligned}
$$

Thus,

$$
\mu z^{1+1 / v-1 / \mu}\left(z^{1 / \mu} f^{\prime}(z)\right)^{\prime}=\frac{1}{v} \int_{0}^{z} w^{1 / v-1} g(w) d w
$$

Making the substitution $w=z s^{v}$ in the above integral and integrating again, a change of variables yields

$$
f^{\prime}(z)=\int_{0}^{1} \int_{0}^{1} g\left(z t^{\mu} s^{v}\right) d s d t
$$


We will use the notation $\phi_{\curlywedge}$ for

$$
\phi_{\lambda}(z)=\int_{0}^{1} \frac{d t}{1-z t^{\lambda}}=\sum_{n=0}^{\infty} \frac{z^{n}}{1+\lambda n}
$$

From [14] it is known that $\phi_{\curlywedge}$ is convex in $U$ provided $\operatorname{Re} \lambda \geq 0$.

Theorem 2.2. Let $\mu$ and $v$ be given by (2.4), and

$$
q(z)=\int_{0}^{1} \int_{0}^{1} h\left(z t^{\mu} s^{v}\right) d t d s
$$

Then the function $q(z)=\left(\phi_{\nu} * \phi_{\mu}\right) * h(z)$ is convex. If $f \in R(\alpha, \gamma, h)$, then

$$
f^{\prime}(z) \prec q(z) \prec h(z)
$$

and $q$ is the best dominant.

Proof. It follows from (2.10) that

$$
h(z) * \phi_{\mu}(z)=\int_{0}^{1} \frac{1}{1-z t^{\mu}} d t * h(z)=\int_{0}^{1} h\left(z t^{\mu}\right) d t:=k(z) .
$$

Thus,

$$
h(z) *\left(\phi_{\mu}(z) * \phi_{v}(z)\right)=k(z) * \phi_{v}(z)=\int_{0}^{1} k\left(z s^{v}\right) d s=\int_{0}^{1} \int_{0}^{1} h\left(z t^{\mu} s^{v}\right) d t d s=q(z) .
$$

Since the convolution of two convex functions is convex [15], the function $q$ is convex. Let

$$
p(z)=f^{\prime}(z)+v z f^{\prime \prime}(z)
$$

Then,

$$
p(z)+\mu z p^{\prime}(z)=f^{\prime}(z)+\alpha z f^{\prime \prime}(z)+\gamma z^{2} f^{\prime \prime \prime}(z) \prec h(z)
$$

It is known from [16] that

$$
p(z) \prec \frac{1}{\mu z^{1 / \mu}} \int_{0}^{z} \zeta^{1 / \mu-1} h(\zeta) d \zeta=\left(\phi_{\mu} * h\right)(z) \prec h(z) .
$$

Similarly,

$$
p(z)=f^{\prime}(z)+v z f^{\prime \prime}(z) \prec\left(\phi_{\mu} * h\right)(z)
$$


implies

$$
\begin{aligned}
f^{\prime}(z) & \prec\left(\phi_{v} * \phi_{\mu} * h\right)(z) \\
& =\sum_{n=0}^{\infty} \frac{z^{n}}{(1+v n)(1+\mu n)} * h(z) \\
& =\left(\int_{0}^{1} \int_{0}^{1} \frac{d t d s}{1-z t^{\mu} s^{v}}\right) * h(z) \\
& =\int_{0}^{1} \int_{0}^{1} h\left(z t^{\mu} s^{v}\right) d t d s=q(z) .
\end{aligned}
$$

The differential chain

$$
f^{\prime} \prec q \prec \phi_{\mu} * h \prec h
$$

shows that $q \prec h$. Since $q(z)+\alpha z q^{\prime}(z)+\gamma z^{2} q^{\prime \prime}(z)=h(z)$, the function

$$
Q(z)=\int_{0}^{z} q(w) d w
$$

is a solution of the differential subordination $f^{\prime}(z)+\alpha z f^{\prime \prime}(z)+\gamma z^{2} f^{\prime \prime \prime}(z)<h(z)$, and thus $q<\tilde{q}$ for all dominants $\tilde{q}$. Hence, $q$ is the best dominant.

Remark 2.3. (1) When $\gamma=0$, then $\mu=0$ and $v=\alpha$, and the above subordination reduces to the result of [16], that is,

$$
f^{\prime}(z)+\alpha z f^{\prime \prime}(z) \prec h(z) \Longrightarrow f^{\prime}(z) \prec \int_{0}^{1} h\left(z t^{\alpha}\right) d t
$$

(2) The above proof also reveals that

$$
f \in R(\alpha, \gamma, h) \Longrightarrow f \in R(0,0, h)
$$

that is, $f^{\prime}(z)<h(z)$.

Theorem 2.4. Let $\mu, v$, and $q$ be as given in Theorem 2.2. If $f \in R(\alpha, \gamma, h)$, then

$$
\begin{aligned}
\frac{f(z)}{z} & \prec \int_{0}^{1} q(t z) d t \\
& =\int_{0}^{1} \int_{0}^{1} \int_{0}^{1} h\left(z r s^{\mu} t^{v}\right) d r d s d t .
\end{aligned}
$$


Proof. Let $p(z)=f(z) / z$. Then

$$
p(z)+z p^{\prime}(z)=f^{\prime}(z) \prec q(z)
$$

With $\phi_{1}$ given by (2.10), this subordination implies

$$
p(z)=\left(\phi_{1} *\left(p+z p^{\prime}\right)\right)(z) \prec\left(\phi_{1} * q\right)(z)=\int_{0}^{1} q(t z) d t
$$

In this paper, starlikeness properties will be investigated for functions $f$ given by a double integral operator of the form (1.3).

\section{Applications}

First, we consider a class of convex univalent functions $h$ so that $h(U)$ is symmetric with respect to the real axis. Denote by $R(\alpha, \gamma, A, B)$ the class

$$
R(\alpha, \gamma, A, B)=\left\{f \in \mathcal{A}: f^{\prime}(z)+\alpha z f^{\prime \prime}(z)+\gamma z^{2} f^{\prime \prime \prime}(z) \prec \frac{1+A z}{1+B z}, z \in U\right\}
$$

where $-1 \leq B<A \leq 1$, and let $h(z ; A, B)=(1+A z) /(1+B z)$. When $A=1-2 \beta$ and $B=-1$, let $h_{\beta}(z):=h(z ; 1-2 \beta,-1)$. The class of $R\left(\alpha, \gamma, h_{\beta}\right)$ is of particular significance, and we will simply denote it by

$$
\begin{aligned}
R\left(\alpha, \gamma, h_{\beta}\right) & :=R(\alpha, \gamma, \beta) \\
& =\left\{f \in \mathcal{A}: f^{\prime}(z)+\alpha z f^{\prime \prime}(z)+\gamma z^{2} f^{\prime \prime \prime}(z) \prec \frac{1+(1-2 \beta) z}{1-z}, z \in U\right\} .
\end{aligned}
$$

Equivalently,

$$
R(\alpha, \gamma, \beta)=\left\{f \in \mathcal{A}: \operatorname{Re}\left(f^{\prime}(z)+\alpha z f^{\prime \prime}(z)+\gamma z^{2} f^{\prime \prime \prime}(z)\right)>\beta\right\}
$$

The following result is an immediate consequence of Theorems 2.2 and 2.4.

Theorem 3.1. Under the assumptions of Theorem 2.2, if

$$
f^{\prime}(z)+\alpha z f^{\prime \prime}(z)+\gamma z^{2} f^{\prime \prime \prime}(z) \prec \frac{1+A z}{1+B z}
$$

then

$$
f^{\prime}(z) \prec \begin{cases}q(z ; A, B) \prec \frac{1+A z}{1+B z}, & \text { if } B \neq 0, \\ q(z ; A) \prec 1+A z, & \text { if } B=0,\end{cases}
$$


where

$$
\begin{gathered}
q(z ; A, B):=1+(A-B) \sum_{n=1}^{\infty} \frac{(-B)^{n-1} z^{n}}{(1+\mu n)(1+v n)} \\
q(z ; A):=1+\frac{A z}{(1+\alpha)}
\end{gathered}
$$

is the best dominant. Further,

$$
\begin{aligned}
\frac{f(z)}{z} & \prec \frac{A}{B}-\frac{A-B}{B} \int_{0}^{1} \int_{0}^{1} \int_{0}^{1} \frac{d s d t d u}{1+B z u t^{\mu} s^{v}} \\
& =1+(A-B) \sum_{n=1}^{\infty} \frac{(-B)^{n-1} z^{n}}{(1+n)(1+\mu n)(1+v n)}
\end{aligned}
$$

if $B \neq 0$, and

$$
\frac{f(z)}{z} \prec 1+\frac{A z}{2(1+\alpha)}
$$

if $B=0$.

\section{Starlikeness Property}

Starlikeness properties of functions given by a double integral operator are investigated in this section. The following result will be required.

Lemma 4.1 (see [5]). If $f \in \mathcal{A}$ satisfies

$$
\operatorname{Re}\left(f^{\prime}(z)+\alpha z f^{\prime \prime}(z)\right)>\frac{(-1 / \alpha) \int_{0}^{1} t^{1 / \alpha-1}((1-t) /(1+t)) d t}{1-1 / \alpha \int_{0}^{1} t^{1 / \alpha-1}((1-t) /(1+t)) d t}, \quad z \in U,
$$

for $\alpha \geq 1 / 3$, then $f \in S^{*}$. This result is sharp.

Theorem 4.2. Let $\mu$ and $v$ be given by (2.4) with $\Delta \geq 0$ and $v \geq 1 / 3$. If

$$
f(z)=\int_{0}^{1} \int_{0}^{1} G\left(z t^{\mu} s^{v}\right) t^{-\mu} s^{-v} d s d t
$$


where $G^{\prime}(z) \prec h_{\beta}(z)=h(z ; 1-2 \beta,-1)$, and $\beta$ satisfies

$$
\beta=1-\frac{1}{2\left(1-(1 / v) \int_{0}^{1} t^{1 / v-1}((1-t) /(1+t)) d t\right)\left(1-\int_{0}^{1}\left(d t /\left(1+t^{\mu}\right)\right)\right)},
$$

then $f \in S^{*}$.

Proof. The function $f$ satisfies

$$
f^{\prime}(z)=\int_{0}^{1} \int_{0}^{1} g\left(z t^{\mu} s^{v}\right) d s d t, \quad G^{\prime}(z)=g(z) \prec h_{\beta}(z)
$$

and thus

$$
f^{\prime}(z)+\alpha z f^{\prime \prime}(z)+\gamma z^{2} f^{\prime \prime \prime}(z)=g(z) \prec h_{\beta}(z)
$$

Now, $\operatorname{Re} h_{\beta}(z)>\beta$ also implies that $\operatorname{Re} g(z)>\beta$, and so

$$
\operatorname{Re}\left(f^{\prime}(z)+\alpha z f^{\prime \prime}(z)+\gamma z^{2} f^{\prime \prime \prime}(z)\right)>\beta, \quad \beta<1 .
$$

It follows from the proof of Theorem 2.2 that

$$
f^{\prime}(z)+v z f^{\prime \prime}(z) \prec\left(\phi_{\mu} * h_{\beta}\right)(z):=q_{\mu}(z)
$$

where

$$
q_{\mu}(z)=2 \beta-1+2(1-\beta) \int_{0}^{1} \frac{d t}{1-z t^{\mu}} .
$$

Since

$$
\operatorname{Re} q_{\mu}(z)>2 \beta-1+2(1-\beta) \int_{0}^{1} \frac{d t}{1+t^{\mu}}
$$

an application of Lemma 4.1 yields the result.

Corollary 4.3. Let $\alpha \geq 3$ and

$$
\operatorname{Re}\left(f^{\prime}(z)+\alpha z f^{\prime \prime}(z)+\frac{\alpha-1}{2} z^{2} f^{\prime \prime \prime}(z)\right)>\beta, \quad \beta<1 .
$$


If $\beta$ satisfies

$$
\beta=1-\frac{1}{2(1-\log 2)\left(1-(2 /(\alpha-1)) \int_{0}^{1} t^{2 /(\alpha-1)-1}((1-t) /(1+t)) d t\right)},
$$

then $f \in S^{*}$.

Proof. In this case, $\mu=1, v=(\alpha-1) / 2$, and the result now follows from Theorem 4.2.

Example 4.4. If

$$
\operatorname{Re}\left(f^{\prime}(z)+3 z f^{\prime \prime}(z)+z^{2} f^{\prime \prime \prime}(z)\right)>\beta
$$

and $\beta$ satisfies

$$
\beta=\frac{4(1-\log 2)^{2}-1}{4(1-\log 2)^{2}} \approx-1.65509
$$

then $f \in S^{*}$.

Theorem 4.5. Let $f, g \in R(\alpha, \gamma, \beta)$ and let $\mu$ and $v$ be given by (2.4) with $\Delta \geq 0$. If $\beta$ satisfies

$$
\beta=1-\frac{1}{4\left(1-\int_{0}^{1} \int_{0}^{1} \int_{0}^{1}\left(d s d t d u /\left(1+u t^{\mu} s^{v}\right)\right)\right)}
$$

then $f * g \in R(\alpha, \gamma, \beta)$.

Proof. Clearly,

$$
(f * g)^{\prime}(z)+\alpha z(f * g)^{\prime \prime}(z)+\gamma z^{2}(f * g)^{\prime \prime \prime}(z)=\left(\left(f^{\prime}+\alpha z f^{\prime \prime}+\gamma z^{2} f^{\prime \prime \prime}\right) * \frac{g}{z}\right)(z) .
$$

Since $f \in R(\alpha, \gamma, \beta)$, substituting $A=1-2 \beta$ and $B=-1$ in (3.7) gives

$$
\operatorname{Re} \frac{g(z)}{z}>2 \beta-1+2(1-\beta) \int_{0}^{1} \int_{0}^{1} \int_{0}^{1} \frac{d s d t d u}{1+u t^{\mu} s^{v}}=\frac{1}{2}
$$

Hence, it follows that

$$
\operatorname{Re}\left((f * g)^{\prime}(z)+\alpha z(f * g)^{\prime \prime}(z)+\gamma z^{2}(f * g)^{\prime \prime \prime}(z)\right)>\beta
$$




\section{Acknowledgments}

The authors are greatly thankful to the referee for the many suggestions that helped improve the presentation of this paper. The work presented here was supported in part by a Research University grant from Universiti Sains Malaysia.

\section{References}

[1] S. S. Miller and P. T. Mocanu, "Double integral starlike operators," Integral Transforms and Special Functions, vol. 19, no. 7-8, pp. 591-597, 2008.

[2] R. M. Ali, "On a subclass of starlike functions," The Rocky Mountain Journal of Mathematics, vol. 24, no. 2, pp. 447-451, 1994.

[3] R. M. Ali and V. Singh, "Convexity and starlikeness of functions defined by a class of integral operators," Complex Variables. Theory and Application, vol. 26, no. 4, pp. 299-309, 1995.

[4] P. N. Chichra, "New subclasses of the class of close-to-convex functions," Proceedings of the American Mathematical Society, vol. 62, no. 1, pp. 37-43, 1977.

[5] R. Fournier and S. Ruscheweyh, "On two extremal problems related to univalent functions," The Rocky Mountain Journal of Mathematics, vol. 24, no. 2, pp. 529-538, 1994.

[6] Y. C. Kim and H. M. Srivastava, "Some applications of a differential subordination," International Journal of Mathematics and Mathematical Sciences, vol. 22, no. 3, pp. 649-654, 1999.

[7] J. Krzyz, "A counter example concerning univalent functions," Folia Societatis Scientiarum Lublinensis, vol. 2, pp. 57-58, 1962.

[8] S. Ponnusamy, "Neighborhoods and Carathéodory functions," Journal of Analysis, vol. 4, pp. 41-51, 1996.

[9] H. Silverman, "A class of bounded starlike functions," International Journal of Mathematics and Mathematical Sciences, vol. 17, no. 2, pp. 249-252, 1994.

[10] R. Singh and S. Singh, "Starlikeness and convexity of certain integrals," Annales Universitatis Mariae Curie-Skłodowska. Sectio A. Mathematica, vol. 35, pp. 145-148, 1981.

[11] R. Szász, "The sharp version of a criterion for starlikeness related to the operator of Alexander," Annales Polonici Mathematici, vol. 94, no. 1, pp. 1-14, 2008.

[12] D.-G. Yang and J.-L. Liu, "On a class of analytic functions with missing coefficients," Applied Mathematics and Computation, vol. 215, no. 9, pp. 3473-3481, 2010.

[13] S. S. Miller and P. T. Mocanu, Differential Subordinations, vol. 225 of Monographs and Textbooks in Pure and Applied Mathematics, Marcel Dekker, New York, NY, USA, 2000.

[14] S. Ruscheweyh, "New criteria for univalent functions," Proceedings of the American Mathematical Society, vol. 49, pp. 109-115, 1975.

[15] S. Ruscheweyh and T. Sheil-Small, "Hadamard products of Schlicht functions and the PolyaSchoenberg conjecture," Commentarii Mathematici Helvetici, vol. 48, pp. 119-135, 1973.

[16] D. J. Hallenbeck and S. Ruscheweyh, "Subordination by convex functions," Proceedings of the American Mathematical Society, vol. 52, pp. 191-195, 1975. 


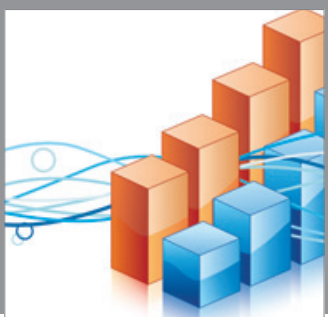

Advances in

Operations Research

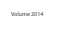

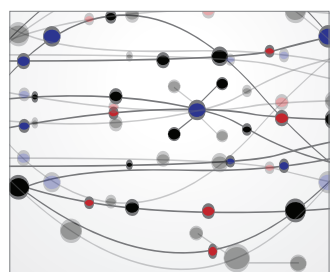

\section{The Scientific} World Journal
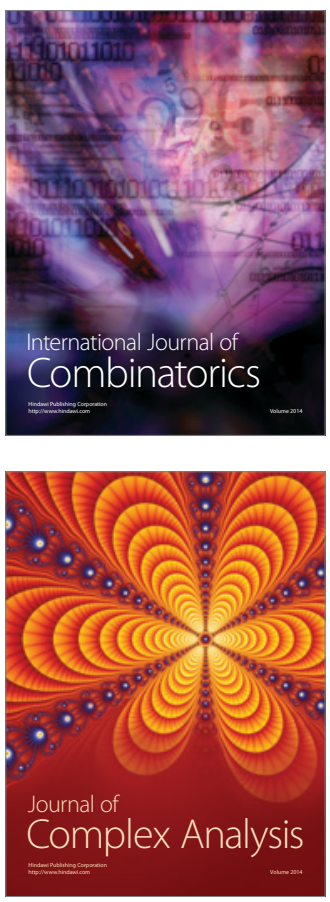

International Journal of

Mathematics and

Mathematical

Sciences
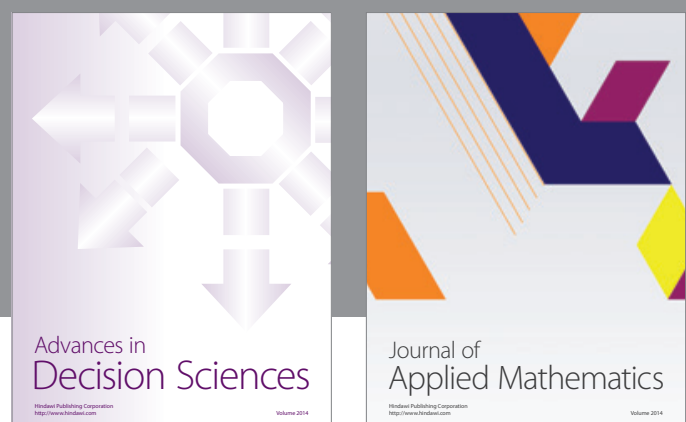

Journal of

Applied Mathematics
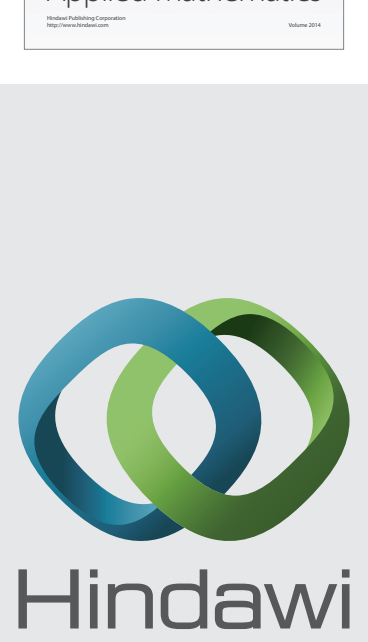

Submit your manuscripts at http://www.hindawi.com
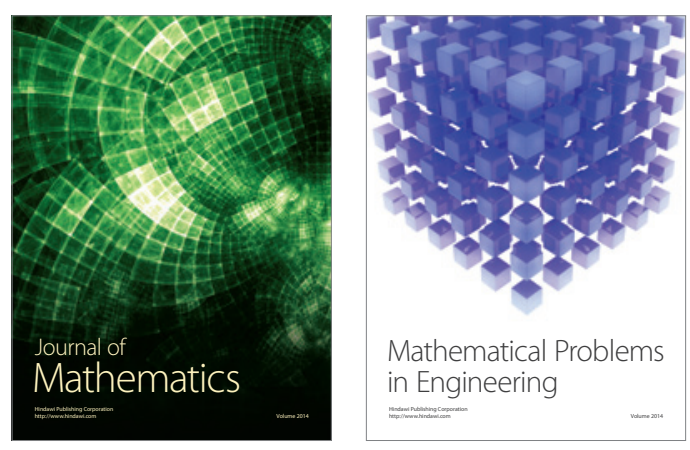

Mathematical Problems in Engineering
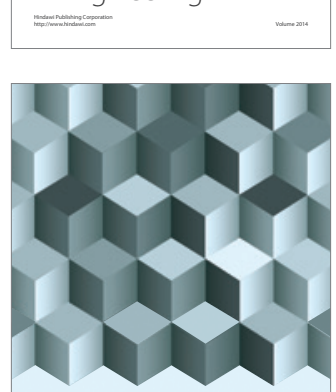

Journal of

Function Spaces
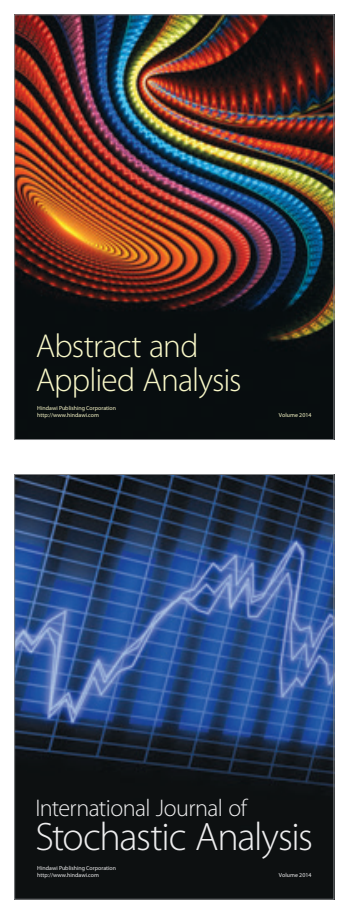

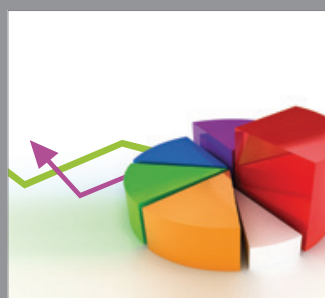

ournal of

Probability and Statistics

Promensencen
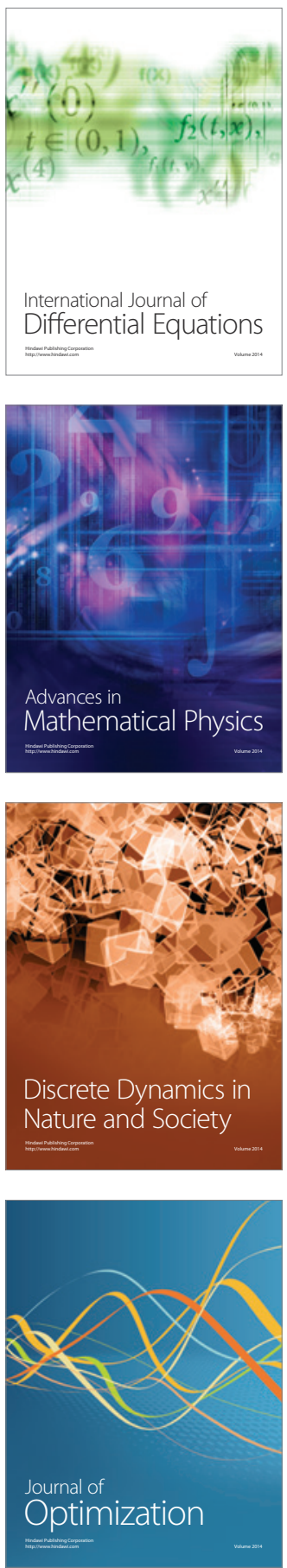